\title{
КОЛИЧЕСТВЕННАЯ ОЦЕНКА РОЛИ СУБИНДЕКСОВ В ФОРМИРОВАНИИ ГЛОБАЛЬНОГО ИННОВАЦИОННОГО ИНДЕКСА
}

\section{QUANTITATIVE ASSESMENT OF THE SUB-INDEXES ROLE IN FORMATION OF GLOBAL INDEX}

F. Mehdi

Summary. Background isin identifying the role of each of two subindexes in the formation of global innovation index. Methodof research is done based on such methods as scientific abstraction and system analysis. Resultisthe conducted research for high- and middle-income countries. Conclusion. Based on cross country data, proposedthe construction of the econometric models.

Keywords: global innovation index, innovation input sub index and product innovation sub index, econometric model.
$\mathbf{K}$ ак известно, одним из наиболее эффективных методов количественной оценки влияния детерминантов на результат является построение регрессионной модели. Для этой цели нами избраны 66 стран: 38 стран с высоким уровнем дохода и $28-$ с уровнем дохода выше среднего по классификации Всемирного Банка. Заметим, что в этой выборке присутствуют, с одной стороны, практически все страны-члены Организации Экономического Сотрудничества и Развития, а с другой - все сопредельные с Азербайджаном страны, кроме Туркменистана (ввиду недоступности определенной информации по этой стране).

Страны с более низкими доходами не включены по двум причинам: во-первых, Азербайджан входит во вторую группу и нацелен на рост ВВП на душу населения; во-вторых, государства с низким уровнем дохода могут создавать ненужные «помехи» при построении эконометрических моделей.

В качестве первичных данных для проведения нашего исследования нами выбраны: а) баллы ГИИ и его субиндексов за 2011, 2019 и 2020 гг. и б) ВВП на душу населения, рассчитанный в соответствии с покупательной способностью относительно текущего курса доллаpa США [1, с. 215-345; 2, c. 218-346; 3, c. 122-244.]

\author{
Мехди Фарид Закир \\ Преподаватель, доктор философии по экономике, \\ докторант, Азербайджанский Государственный \\ Университет Нефти и Промышленности, г. Баку \\ farid.mehdi2@gmail.com
}

Аннотация. Цель работы заключается в выявлении роли каждого из двух субиндексов в формировании глобального инновационного индекса. Исследование основывается на таких методах, как научная абстракция и системный анализ. Вывод сделан на основе результатов проведённых исследований для стран с высоким и средним уровнем дохода. На основе перекрестных данных по странам предложено построение эконометрических моделей.

Ключевые слова: глобальный инновационный индекс, субиндекс ввода инноваций и субиндекс инновационной продукции, эконометрическая модель.

Прежде всего, представляет интерес степень вклада детерминантов ГИИ на его значение. Мы руководствуемся оценкой вклада двух субиндексов. Во-первых, потому что выводы, сделанные на более агрегированном уровне, иногда могут быть полезнее для принятия политических решений. Второй аргумент в пользу такого выбора не менее интересен, и он учитывается в нашей методике, которая имеет ряд следующих особенностей:

1. Дело в том, что по определению вклад этих субиндексов в значение ГИИ должен быть одинаков, так как Глобальный инновационный индекс вычисляется как их среднее арифметическое значение. Поэтому в качестве роли субиндексов мы не рассматриваем их непосредственный вклад ГИИ. Мы будем генерировать модели, в которых регрессантом является рост ГИИ, а регрессорами - рост значений субиндексов.

2. В данной работе рассматривается период с 2011 по 2019 год. Анализ данных за 2020 год показывает, что они не имеют практической ценности в виду пандемии COVID-19 в глобальном масштабе. Как показал наш анализ, практически по всем странам сократились не только экономические показатели (в частности, ВВП на душу населения), но и сам уровень работы в области внедрения инноваций. Например, у первой пятерки 
Таблица 1. Модель зависимости глобального инновационного индекса от значений его субиндексов ввода инноваций и инновационной продукции для всей выборки

\begin{tabular}{|c|c|c|c|c|}
\hline \multicolumn{3}{|c|}{ Dependent Variable: GII } & & \\
\hline \multicolumn{5}{|c|}{ Method: Least Squares (Gauss-Newton / Marquardt steps) } \\
\hline \multicolumn{3}{|c|}{ Date: 08/11/21 Time: 12:12 } & & \\
\hline \multicolumn{2}{|l|}{ Sample: 166} & & & \\
\hline \multicolumn{5}{|c|}{ Included observations: 66} \\
\hline \multicolumn{5}{|c|}{$\mathrm{G} \|=\mathrm{C}(1)+\mathrm{C}(2)^{*} \mathrm{INPUT}+\mathrm{C}(3)^{*}$ OUTPUT } \\
\hline & Coefficient & Std. Error & t-Statistic & Prob. \\
\hline$C(1)$ & -0.082025 & 0.400208 & -0.204956 & 0.8383 \\
\hline$C(2)$ & 0.543487 & 0.036205 & 15.01138 & 0.0000 \\
\hline$C(3)$ & 0.339021 & 0.017096 & 19.83038 & 0.0000 \\
\hline R-squared & 0.909355 & \multicolumn{2}{|c|}{ Mean dependent var } & 3.635000 \\
\hline Adjusted R-squared & 0.906478 & \multicolumn{2}{|c|}{ S.D. dependent var } & 7.405121 \\
\hline S.E. of regression & 2.264592 & \multicolumn{2}{|c|}{ Akaike info criterion } & 4.517056 \\
\hline Sum squared resid & 323.0879 & \multicolumn{2}{|c|}{ Schwarz criterion } & 4.616586 \\
\hline Log likelihood & -146.0628 & \multicolumn{2}{|c|}{ Hannan-Quinn criter. } & 4.556385 \\
\hline F-statistic & 316.0102 & \multicolumn{2}{|c|}{ Durbin-Watson stat } & 1.634019 \\
\hline Prob(F-statistic) & 0.000000 & & & \\
\hline
\end{tabular}

Таблица сгенерирована автором с использованием программы Eviews

стран-лидеров по ГИИ в 2020 оду индекс значительно снизился.

3. В Швейцарии ГИИ упал на 1,73\%, субиндекс ввода инноваций - на 2,25\%, субиндекс производства инновационной продукции - на 1,10\%, а ВВП на душу населения - на 10,61\%, в Швеции, соответственно, на 1,85\%, 1,76\%, 1,97\% и 10\%, в США - на 1,9\%, 2,84\%, 0,63\% и 9,2\%, в Объединенном Королевстве - на 2,48\%, 3,3\%, 1,45\% и 10,55\%, в Нидерландах - на 4,36\%,1,45\%, 7,67\% и 9,67\%. В Азербайджане наблюдалось ещё более масштабное снижение этих показателей: ГИИ упал на 9,86\%, субиндекс ввода инноваций - на 5,82\%, субиндекс выхода инновационной продукции - на 18,8\%, а ВВП на душу населения - на 10,09\%. Интересно то, что уменьшения по всем этим индикаторам имели место во всех выбранных нами странах, кроме Мавритании, ГИИ которой увеличился на 12,22\%, субиндекс ввода инноваций - на 5,83\%, субиндекс выхода инновационной продукции - на 27,73\%, хотя ВВП на душу населения в этой стране сократился на $7,92 \%[1$, с. 7$]$.

Наконец, отметим, что найденные нами значения коэффициентов этих субиндексов в регрессионной модели будут использоваться для оценки их «реального» усредненного вклада в ГИИ выбранных стран.

\section{1. Обшая модель по рассматриваемым странам}

Итак, общий результат, полученный при построении регрессионной модели по всем 66 странам, представлен в таблице 1. Как видно, статистические характеристики модели, сгенерированной на основе критерия наименьших квадратов, хорошие, т.е. полученная модель 1 адекватна.

$$
\text { GII }=-0,08+0,54 * \text { INPUT }+0,34^{*} \text { OUTPUT }
$$

Здесь GII - глобальный инновационный индекс, INPUT - значение субиндекса ввода инноваций, OUTPUT - значение субиндекса инновационной продукции.

Основной признак адекватности модели заключается в достаточно высокой степени значимости коэффициентов регрессоров, так как очень низкие значения обоих коэффициентов указывает на то, что их значимость не слишком высока [2, с. 26]. С другой стороны, коэффициент детерминации имеет достаточно высокое значение: $\mathrm{R}^{2}=0,91$. Значение статистики Дарбина-Уатсона DW = 1,63 указывает на незначительную положительную автокорреляцию. Заметим так же, что сумма квадратов отклонений RSS = 323 (табл. 4.1.1) тоже хорош, если учесть, что: 
Таблица 2. Модель зависимости ГИИ от значений его субиндексов для стран с высоким уровнем дохода

\begin{tabular}{|c|c|c|c|c|}
\hline \multicolumn{3}{|c|}{ Dependent Variable: GII } & & \\
\hline \multicolumn{5}{|c|}{ Method: Least Squares (Gauss-Newton / Marquardt steps) } \\
\hline \multicolumn{5}{|c|}{ Date: 08/12/21 Time: 19:26 } \\
\hline \multicolumn{5}{|l|}{ Sample: 138} \\
\hline \multicolumn{5}{|c|}{ Included observations: 38} \\
\hline \multicolumn{5}{|c|}{$\mathrm{G} \|=\mathrm{C}(1)+\mathrm{C}(2)^{*} \mathrm{INPUT}+\mathrm{C}(3)^{*}$ OUTPUT } \\
\hline & Coefficient & Std. Error & t-Statistic & Prob. \\
\hline$C(1)$ & 0.289230 & 0.413437 & 0.699573 & 0.4888 \\
\hline$C(2)$ & 0.564490 & 0.052456 & 10.76120 & 0.0000 \\
\hline$C(3)$ & 0.359634 & 0.030847 & 11.65849 & 0.0000 \\
\hline R-squared & 0.892404 & \multicolumn{2}{|l|}{ Mean dependent var } & 3.743158 \\
\hline Adjusted R-squared & 0.886256 & \multicolumn{2}{|l|}{ S.D. dependent var } & 5.526721 \\
\hline S.E. of regression & 1.863943 & \multicolumn{2}{|l|}{ Akaike info criterion } & 4.158922 \\
\hline Sum squared resid & 121.6000 & \multicolumn{2}{|l|}{ Schwarz criterion } & 4.288205 \\
\hline Log likelihood & \begin{tabular}{|l|}
-76.01952 \\
\end{tabular} & \multicolumn{2}{|l|}{ Hannan-Quinn criter. } & 4.204920 \\
\hline F-statistic & 145.1453 & \multicolumn{2}{|l|}{ Durbin-Watson stat } & 0.960055 \\
\hline Prob(F-statistic) & 0.000000 & & & \\
\hline
\end{tabular}

Таблица сгенерирована автором с использованием программы Eviews

а) среднее значение зависимой переменной $\mathrm{E}(\mathrm{GII})=$ 3,64 (табл. 4.1.1);

б) RSS представляет собой сумму для 66 наблюдений;

в) RSS - сумма не простых чисел, а квадратов.

Интерпретируя модель, можем утверждать, что увеличение процентного роста субиндекса ввода инноваций на один пункт приводит к росту ГИИ на 0,54 пункта, а увеличение процентного роста субиндекса выхода инновационной продукции на один процентный пункт — к увеличению процентного роста ГИИ на 0,34 пункта.

Итак, модель 1 указывает на то, что для используемой нами достаточно представительной выборки влияние на ГИИ субиндекса ввода инноваций, больше, чем субиндекса инновационной продукции. Данный факт подтверждается двумя гипотезами, к которым мы и склоняемся.

Гипотеза 1. В выбранных странах основное внимание уделяется стимулированию инновационной деятельности, а не ее эффективности.

Гипотеза 2. Такой результат в определенном смысле естественен по следующчим объективным причинам.

- Во-первых, несколько организаций (компаний) могут осуществлять инновационную деятельность в одном и том же направлении, но регистрируется результат, достигнутый только одной из них.
- Во-вторых, для успеха инновационной деятельности требуются годы, при этом не каждый проект может увенчаться успехом.

- Наконец, фундаментальные исследования (в большинстве своем финансируемые государством) фиксируются как вводная часть инновационной деятельности, но их результатом становится исключительно узконаправленный эффект.

Как будет видно из последующих сравнений, полезно вводить понятие относительной эффективности субиндексов. Для этой цели, не умаляя роли входных факторов инноваций, заметим, что, в конечном счете, пользу для экономического и социального развития страны приносят выходные индикаторы, т.е. конечная инновационная продукция. Поэтому в качестве относительной эффективности субиндексов примем отношение коэффициента OUTPUT к коэффициенту INPUT:

$$
E f=C(3) / C(2)
$$

Таким образом, этот показатель для смешанной выборки $\mathrm{Ef} \approx 0,34 / 0,54 \approx 0,624$.

\section{2. Модель по странам \\ с высоким уровнем $\triangle O х о \Delta а$}

Имеет смысл рассмотреть, как влияние субиндексов на ГИИ меняется при раздельном исследовании развитых и только развивающихся стран. В таблице 2 отражена модель для 38 стран с высоким уровнем дохода, а ее уравнение приводится в таблице 3. Несмотря на то, что 
Таблица 3. Модель зависимости ГИИ от значений его субиндексов для стран с уровнем дохода выше среднего

\begin{tabular}{|c|c|c|c|c|}
\hline \multicolumn{3}{|c|}{ Dependent Variable: GII } & & \\
\hline \multicolumn{5}{|c|}{ Method: Least Squares (Gauss-Newton / Marquardt steps) } \\
\hline \multicolumn{3}{|c|}{ Date: 08/12/21Time: 19:27 } & & \\
\hline \multicolumn{2}{|l|}{ Sample: 128} & & & \\
\hline \multicolumn{3}{|c|}{ Included observations: 28} & & \\
\hline \multicolumn{4}{|c|}{$G \|=C(1)+C(2)^{*} \mid N P U T+C(3)^{*}$ OUTPUT } & \\
\hline & Coefficient & Std. Error & t-Statistic & Prob. \\
\hline$C(1)$ & -1.331955 & 0.825573 & -1.613371 & 0.1192 \\
\hline$C(2)$ & 0.585185 & 0.057147 & 10.23990 & 0.0000 \\
\hline$C(3)$ & 0.321646 & 0.022776 & 14.12237 & 0.0000 \\
\hline R-squared & 0.930494 & \multicolumn{2}{|c|}{ Mean dependent var } & 3.488214 \\
\hline Adjusted R-squared & 0.924933 & \multicolumn{2}{|c|}{ S.D. dependent var } & 9.492937 \\
\hline S.E. of regression & 2.600908 & \multicolumn{2}{|c|}{ Akaike info criterion } & 4.850555 \\
\hline Sum squared resid & 169.1181 & \multicolumn{2}{|c|}{ Schwarz criterion } & 4.993292 \\
\hline Log likelihood & -64.90778 & \multicolumn{2}{|c|}{ Hannan-Quinn criter. } & 4.894191 \\
\hline F-statistic & 167.3394 & \multicolumn{2}{|c|}{ Durbin-Watson stat } & 1.902515 \\
\hline Prob(F-statistic) & 0.000000 & & & \\
\hline
\end{tabular}

Таблица сгенерирована автором с использованием программы Eviews

коэффициент детерминации в данной модели немного ниже, а автокорреляция существенно выше, она позволяет объективно рассмотреть статистические данные: коэффициенты переменных значимы, да и коэффициент детерминации достаточно высок [3, с. 390].

$$
\mathrm{GII}=0,30+0,57^{*} \mathrm{INPUT}+0,36^{*} \text { OUTPUT }
$$

3. Влияние вкладов субиндексов на рост ГИИ аналогичен с предыдущей моделью, и, хотя в абсолютном выражении коэффициент ввода инноваций вырос больше, чем коэффициент инновационной продукции, относительная эффективность субиндексов здесь немного больше: $\mathrm{Ef} \approx 0,36 / 0,57 \approx 0,637$. А это означает, что при совокупном рассмотрении выборки из всех стран ГИИ развивающихся стран имеет тенденцию к понижению.

\section{3. Молель по странам \\ с уровнем $\triangle$ охола выше срелнего}

Как видно из таблицы 3, схожая модель, сгенерированная для стран с уровнем дохода выше среднего, имеет вид, описанный формулой (4). Эта модель более эффективна по сравнению с предыдущими: наряду с высоко значимыми коэффициентами переменных, ее коэффициент детерминации $\mathrm{R}^{2}$ составляет 0,93, то есть он самый высокий. С другой стороны, автокорреляция практически отсутствует: статистика Дарбина-Уатсона $\mathrm{DW}=1,9$.

$$
\text { GII }=-1,33+0,59 * \text { INPUT }+0,32 * \text { OUTPUT }
$$

Относительная роль субиндексов в росте ГИИ, по сути, не изменилась, но существенно уменьшилась относительная эффективность субиндексов выражается как $\mathrm{Ef} \approx 0,32 / 0,59 \approx 0,550$, что почти на $14 \%$ аналогичного показателя стран с высоким уровнем дохода.

В смысле соотношения выходной части и вводных данных, инновационная деятельность (точнее говоря, инновационные усилия) более эффективна в развитых, нежели в развивающихся странах.

Мы рассчитали вклады двух субиндексов в рост ГИИ. Но предложенная методика может быть применена для оценки вклада семи отдельных компонентов субиндексов, а также для 80 общих субиндикаторов. 


\section{ЛИТЕРАТУРА}

1. The Global Innovation Index 2020. Who Will Finance Innovation? Editors: Dutta S, Lanvin B., Wunsch-Vincent S. — Cornell University, INSEAD, and the World Intellectual Property Organization, 2020.— $448 \mathrm{p}$.

2. Thomas J., Huggett S., Kamalski J. Finding a Way Through the Scientific Literature: Indexes and Measures // World Neurosurgery, 2011, Vol. 76, № 1-2, pp. 36-38.

3. The Global Innovation Index 2011 Accelerating Growth and Development. Editor: Dutta S., INSEAD, 2011. - $381 \mathrm{p}$.

4. Global innovation index 2019. Creating healthy lives — the future of medical innovation. Editors: Dutta S, Lanvin B., Wunsch-Vincent S. — INSEAD, 2019. $500 \mathrm{p}$.

5. The Bio Revolution: Innovations transforming economies, societies, and our lives. — McKinsey Global Institute, 2020.— $200 \mathrm{p}$.

6. Business innovation statistics and indicators / https://www.oecd.org/innovation/inno-stats.htm\#definitions

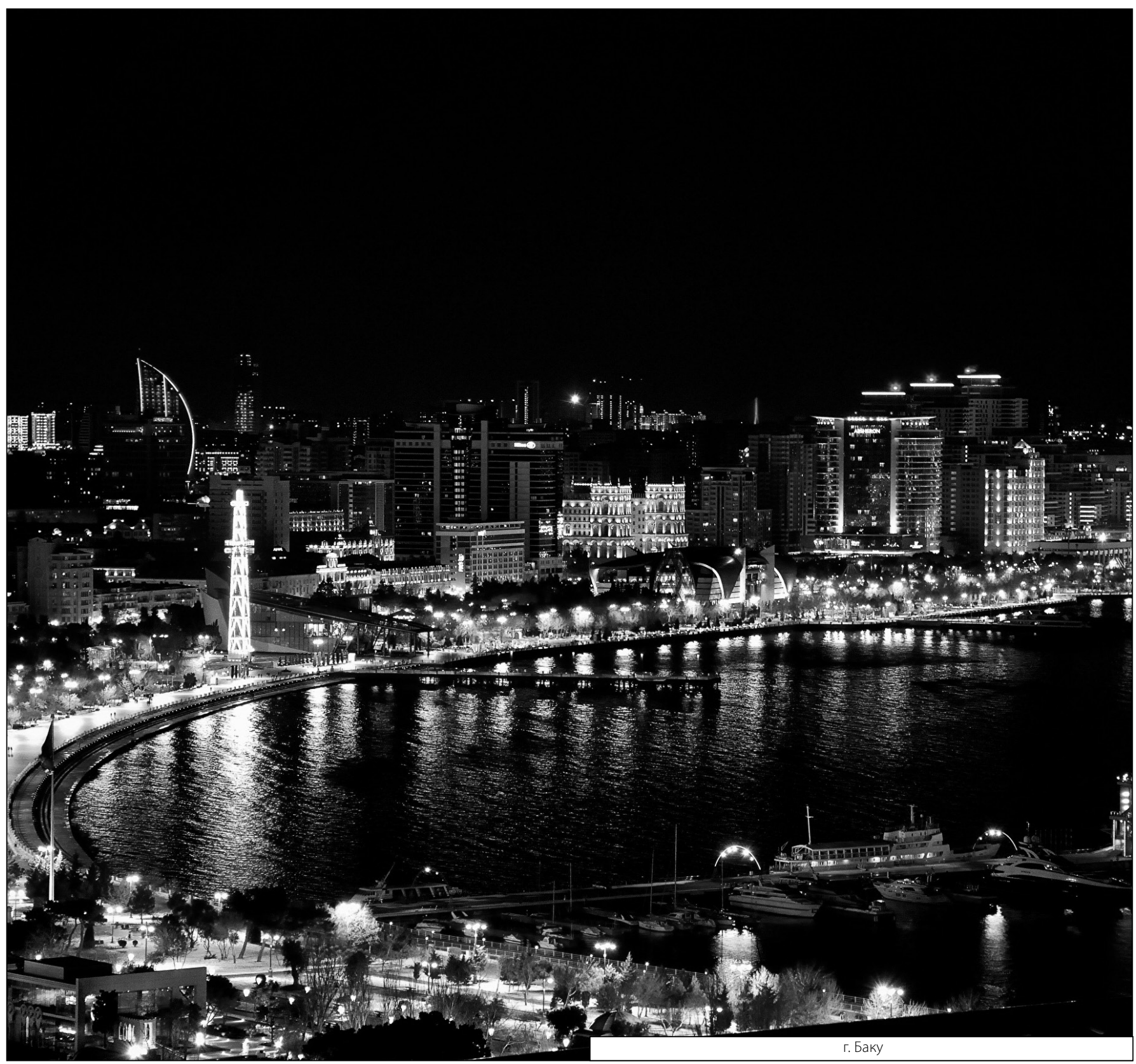

